\title{
Apoptotic response of eosinophils in chronic eosinophilic pneumonia
}

\author{
N. Saita*, T. Yamanaka*, H. Kohrogi*, M. Ando*, M. Hirashima*
}

\begin{abstract}
Apoptotic response of eosinophils in chronic eosinophilic pneumonia. N. Saita, T. Yamanaka, M. Ando, M. Hirashima. C) ERS Journals Ltd 2001.

ABSTRACT: To clarify the pathogenesis of chronic eosinophilic pneumonia (CEP), the apoptosis of eosinophils from bronchoalveolar lavage (BAL-Eos) was compared with that of eosinophils from peripheral blood (PB-Eos) in six cases of CEP.

The survival rate of eosinophils and the percentage of apoptotic cells of both types of eosinophils were examined, and the effects of interleukin 5 (IL-5) were evaluated. The role of Fas expression in apoptosis of these eosinophils was also studied.

The survival rate of BAL-Eos on the third day of culture was significantly higher than that of PB-Eos $(\mathbf{p}<\mathbf{0 . 0 1})$. This was associated with a lower proportion of apoptotic cells in BAL-Eos than in PB-Eos; the percentages of apoptotic cells in PB-Eos and BAL-Eos after $24 \mathrm{~h}$ of incubation were $21.7 \pm 3.4 \%$ and $10.6 \pm 1.7 \%$ respectively. IL-5 suppressed apoptosis and increased the survival rate of both PB-Eos and BAL-Eos. It was found that the apoptotic character of BAL-Eos differed from that of PB-Eos in at least three ways. Firstly, the positive rate of Fas expression on PB-Eos was increased after $24 \mathrm{~h}$ of incubation, whereas that on BAL-Eos did not change. Secondly, the expression of Fas on PB-Eos was suppressed by IL-5 $(18.5 \pm 4.2 \%-8.3 \pm 3.2 \%, p<0.05)$, whereas IL-5 failed to suppress Fas expression on BAL-Eos $(3.3 \pm 1.6 \%-3.6 \pm 1.0 \%)$. Lastly, binding of antibody to Fas antigen induced apoptosis of PB-Eos, but not of BAL-Eos.

These data suggested that Fas seemed to be involved in the apoptosis of PB-Eos, whereas BAL-Eos were Fas-resistant in chronic eosinophilic pneumonia. In conclusion, apoptosis of eosinophils might be suppressed by proinflammatory cytokines such as IL-5 leading to their accumulation in the lung. Chronic stimulation of eosinophils in the alveolar space with IL-5 may play a crucial role chronic eosinophilic disorders. Eur Respir J 2001; 17: 190-194.
\end{abstract}

\begin{abstract}
*First Dept of Internal Medicine, Kumamoto University, School of Medicine, Kumamoto, Japan and \#Dept of Immunology and Immunopathology, Kagawa Medical School, Kagawa, Japan
\end{abstract}

Correspondence: N. Saita, First Dept of Internal Medicine, Kumamoto University, School of Medicine, 1-1-1 Honjo, Kumamoto, 860-0811, Japan Fax: 01181963710582

Keywords: Chronic eosinophilic pneumonia eosinophil apoptosis interleukin-5

Received: November 31999 Accepted after revision September 5 2000
Chronic eosinophilic pneumonia (CEP) is characterized by the presence of an interstitial eosinophilic inflammatory reaction in the lung, and a large number of eosinophils in the bronchoalveolar lavage (BAL-Eos) [1]. An increased production of interleukin (IL)-5 seems to be one of the critical pathophysiological features of eosinophilic pneumonia [2, 3]. Several reports have shown that IL-5 was present in BAL fluid (BALF), and the level of IL-5 was reduced after resolution of pulmonary eosinophilia $[4,5]$.

The viability of eosinophils in the peripheral blood (PB-Eos) can be maintained in culture for several days and prolonged by IL-5, and these findings have been interpreted as implying that IL-5 delays apoptosis and prolongs the survival of eosinophils [6]. However, the precise interaction between eosinophils and IL-5 in the alveolar space is not fully understood. Although several studies have been performed on the correlation between eosinophils and apoptosis [7-9], there have been few comparisons of apoptosis of eosinophils from PB and BAL in CEP. Other reports have shown that the decreased or suppressed apoptosis of eosinophils in nasal polyps and the bronchi of bronchial asthma patients is an important mechanism for the persistence of chronic specific eosinophil accumulation $[10,11]$. It is also suspected that a decrease of apoptosis might also be present in the pulmonary parenchyma in CEP.

The purpose of this study was to determine whether eosinophil apoptosis is suppressed in the alveolar space in CEP. The following were evaluated: 1) the kinetics of the apoptosis of PB- and BAL-Eos from CEP; 2) the effect of IL-5 on apoptosis in these cells; and 3) the involvement of Fas expression.

\section{Materials and methods}

\section{Patients}

The diagnosis of CEP was based on the patient's past history, physical examination, radiographic features, and the presence of increased numbers of eosinophils in lung tissue and BAL. As shown in table 1, six cases of CEP were examined. None of the patients received steroid therapy before obtaining PB-Eos and BAL-Eos.

\section{Cell fractionation}

Peripheral blood leukocytes were obtained from heparinized venous blood. The separation of eosinophils was performed using a discontinuous density Percoll 
Table 1.-Cell number and percentage of eosinophils from peripheral blood and bronchoalveolar lavage in six cases of chronic eosinophilic pneumonia

\begin{tabular}{|c|c|c|c|c|c|c|}
\hline \multirow{2}{*}{ Case } & \multirow{2}{*}{ Age } & \multirow{2}{*}{ Sex } & \multicolumn{2}{|c|}{ Eosinophil from peripheral blood } & \multicolumn{2}{|c|}{ Eosinophil from bronchoalveolar lavage } \\
\hline & & & Number $\cdot \mathrm{mm}^{-3}$ & $\%$ & Number $1 \times 10^{5} \cdot \mathrm{mL}^{-1}$ & $\%$ \\
\hline 1 & 56 & $\mathrm{~F}$ & 795 & 17.1 & 1.23 & 25.3 \\
\hline 2 & 43 & $\mathrm{~F}$ & 623 & 15.2 & 1.74 & 14.5 \\
\hline 3 & 45 & $\mathrm{~F}$ & 754 & 13.0 & 11.63 & 40.8 \\
\hline 4 & 41 & $\mathrm{~F}$ & 2297 & 23.2 & 2.30 & 23.0 \\
\hline 5 & 57 & M & 2871 & 29.0 & 2.73 & 26.5 \\
\hline 6 & 60 & M & 2720 & 32.7 & 14.30 & 40.7 \\
\hline
\end{tabular}

column (Pharmacia, Uppsala, Sweden) with five different concentrations, as previously described [12]. To remove residual neutrophils, a monoclonal antibody against CD 16 in a negative, indirect selection procedure using imunomagnetic beads (Dynal AS, Oslo, Norway) was used. BAL was performed in the involved lung segment. BALF was centrifuged at 1,200 revolutions per minute (rpm) for $10 \mathrm{~min}$. Eosinophils from BAL were also enriched with a discontinuous density Percoll column, and the cells were suspended in $10 \%$ foetal calf serum-containing RPMI 1640 solution. In all cases, the eosinophil-rich fraction was $>98 \%$ pure, and the viability was $>99 \%$.

\section{Evaluation of eosinophil survival and apoptosis}

The viability of the eosinophils was evaluated by the trypan blue dye exclusion test after 3 days of incubation at $37^{\circ} \mathrm{C}$ in a $5 \% \mathrm{CO}_{2}$-containing incubator. The effects of IL-5 on eosinophil survival were also assessed. Recombinant human IL-5 (10 ng. $\mathrm{mL}^{-1}$, Gibco BRL, Grand Island, NY, USA) was used for stimulation. In some cases, the kinetics of viability were examined after $1,3,5$, and 7 days of incubation. Apoptosis of the eosinophils was assessed with an annexin-V FITC Kit (Immunotech, Marseille Cedex, France) according to the methods of Levi-SchafFER et al. [13]. Briefly, eosinophils were cultured for $24 \mathrm{~h}$ in the presence or absence of IL-5 (10 ng. $\left.\mathrm{mL}^{-1}\right)$, harvested and washed with phosphate-buffered saline by centrifugation for $3 \mathrm{~min}$ at 4,000 rpm. The cells were resuspended in $490 \mu \mathrm{L}$ of binding buffer $(10 \mathrm{mM}$ Hepes, $140 \mathrm{mM}$ $\mathrm{NaCl}, 2.5 \mathrm{mM} \mathrm{CaCl}_{2}, \mathrm{pH} 7.4$ ), and $1.25 \mu \mathrm{g}$ propidium iodide and $5 \mu \mathrm{L}$ of annexin-V FITC solution were added. After a 10-min incubation on ice, the cell sample was analysed using a FACScan flow cytorneter (Becton Dickinson, Mountain View, CA, USA).

The effects of IL-5 on Fas expression on the eosinophils were also assessed. PB-Eos and BAL-Eos were cultured for $24 \mathrm{~h}$, and then the harvested cells were incubated with monoclonal antibodies against Fas (UB2, MBL, Nagoya, Japan) for $30 \mathrm{~min}$. After washing, the cells were analysed using the FACScan flow cytometer.

In some cases, PB-Eos and BAL-Eos were cultured for $24 \mathrm{~h}$ with or without monoclonal antibodies against Fas (CH11, MBL, Nagoya, Japan; $\left.300 \mathrm{ng} \cdot \mathrm{mL}^{-1}\right)$ for induction of Fas-induced apoptosis. Apoptosis was assessed with an annexin-V FITC Kit, as described above.

\section{Statistical analysis}

Results are expressed as the mean \pm standard deviation (SD). Differences between the groups were assessed by the Mann-Whitney test. A p-value $<0.05$ was considered to have statistical significance.

\section{Results}

In order to elucidate the survival rates of PB-Eos and BAL-Eos, they were cultured for 3 days, and the viability was determined by trypan blue dye exclusion. Figure 1 shows that the survival rate of BAL-Eos was significantly higher than that of PB-Eos with individual paired data $(\mathrm{p}<0.01)$. The effect of IL-5 on the survival rates of both cells was examined. The percentage of viable cells in IL-5-treated PB-Eos significantly increased $1.3+1.2 \%-21.7+5.8 \%$, and that of IL-5-treated BAL-Eos also significantly increased $39.1 \pm 10.9 \%-81.0 \pm 14.5 \% \quad$ (fig. $1 \mathrm{a} ; \quad \mathrm{p}<0.05$ and $\mathrm{p}<0.05$, respectively). Such survival-enhancing effects of IL-5 on both type of eosinophils were still detected after 7 days of incubation (figs. $1 b$ and c). As the effect of IL-5 on eosinophil survival was dose dependent up to $10 \mathrm{ng} \cdot \mathrm{mL}^{-1}$, this optimal dose was used in all experiments.

The effect of IL-5 on eosinophil apoptosis was further assessed. PB-Eos and BAL-Eos were cultured in the presence or absence of IL-5 for $24 \mathrm{~h}$. The percentages of apoptotic cells in PB-Eos and BALEos were $21.7 \pm 3.4 \%$ and $10.6 \pm 1.7 \%$, respectively (fig. 2), suggesting that BAL-Eos were more resistant to apoptosis than PB-Eos $(\mathrm{p}<0.05)$. It was also found that IL-5 significantly suppressed apoptosis in both types of cells. When PB-Eos and BAL-Eos were cultured with IL-5, the percentage of apoptotic PB-Eos decreased from $21.7 \pm 3.4 \%$ to $12.2 \pm 2.0 \%$, and that of apoptotic BAL-Eos decreased from $10.6 \pm 1.7 \%$ to $5.2 \pm 1.1 \%$ ( $\mathrm{p}<0.05$ for both) (fig. 2).

Although IL-5 suppressed apoptosis of both PB- and BAL-Eos, the mechanism of apoptosis in the two types of eosinophils was suggested to be different in several ways: the expression of Fas antigen on PB-Eos increased significantly from $7.8 \pm 1.8 \%$ to $18.5 \pm 4.2 \%$ after $24 \mathrm{~h}$ of incubation $(\mathrm{p}<0.05)$, whereas that on BAL-Eos did not (from $5.2 \pm 1.9 \%$ to $3.3 \pm 1.6 \%$ ) (fig. 3). The effect of IL-5 on Fas expression on eosinopilils after $24 \mathrm{~h}$ of incubation was also different in the two types of eosinophils. As shown in figure 3a, the Fas expression of PB-Eos was significantly 

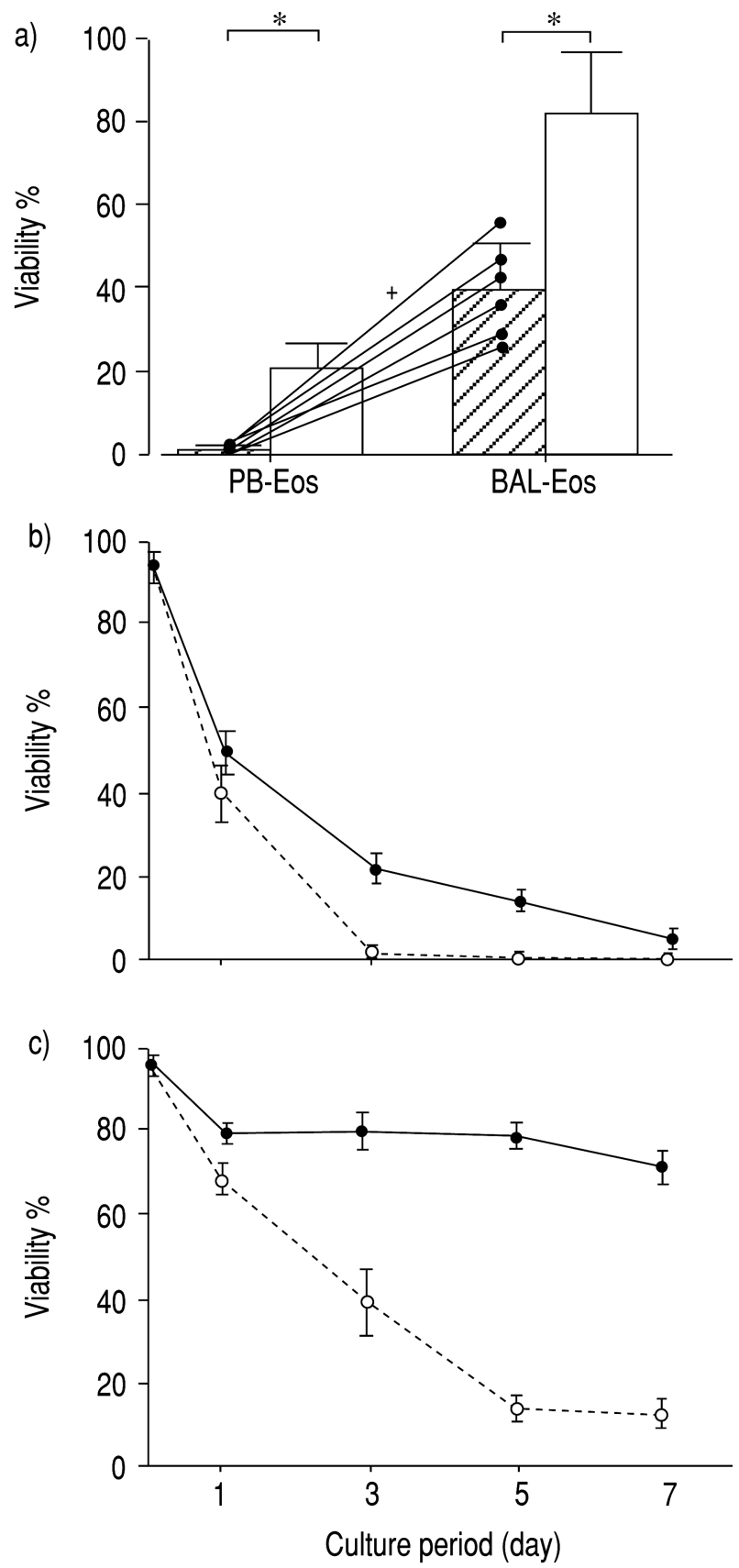

Fig. 1. - Effect of interleukin (IL)-5 on the viability of eosinophils from peripheral blood (PB-Eos) and eosinophils from bronchoalveolar lavage (BAL-Eos). a) PB-Eos and BAL-Eos of chronic eosinophilic pneumonia (CEP) patients were cultured with or without IL-5 (10 ng $\left.\cdot \mathrm{mL}^{-1}\right)$ for 3 days and harvested. The viability of the eosinophils was evaluated by the trypan blue dye exclusion test. Data represented with individual paired data when grown in phosphate buffered saline (PBS) with lines for each of the six patients. $\nabla$ : PBS; $\square$ : IL-5. Other data represent the means \pm SD of six cases. Time-courses of the viability of b) PB-Eos and c) BAL-Eos with $(\bullet)$ or without IL-5 $(O)$ were evaluated. Representative data from one patient is shown. Data represent the mean $\pm \mathrm{SD}$ of triplicate cultures. ${ }^{*}: \mathrm{p}<0.05$; $^{+}$: $\mathrm{p}<0.01$.

suppressed by co-culturing with IL-5 (from $18.5 \pm 4.2 \%$ to $8.3 \pm 3.2 \%, \mathrm{p}<0.05)$, whereas that of BAL-Eos was not suppressed (fig. 3b).
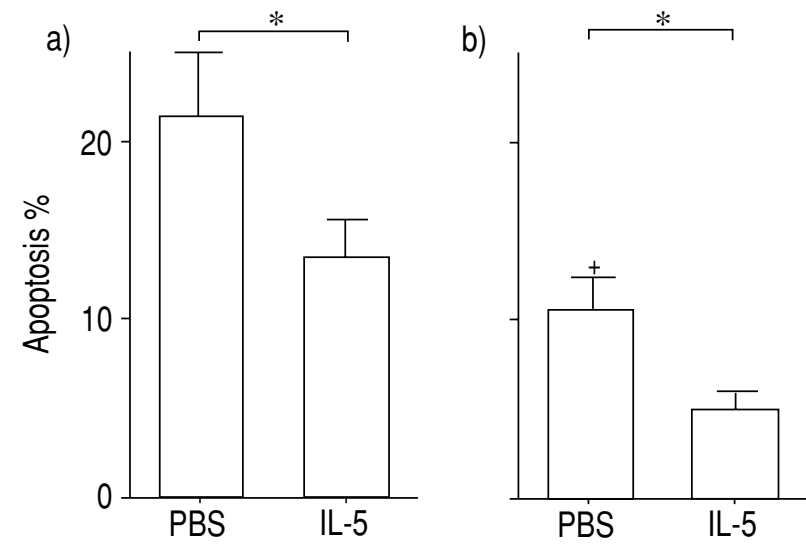

Fig. 2. - Effect of interleukin (IL)-5 on the apoptosis of eosinophils from peripheral blood (PB-Eos) and eosinophils from bronchoalveolar lavage (BAL-Eos). Eosinophils from a) peripheral blood (PB) and b) bronchoalveolar lavage (BAL) were cultured for $24 \mathrm{~h}$. Data are the mean + SD of six cases. *. $\mathrm{p}<0.05$; $^{+}: \mathrm{p}<0.05$ versus $\mathrm{PB}-\mathrm{E}$ os treated with phosphate buffered saline.
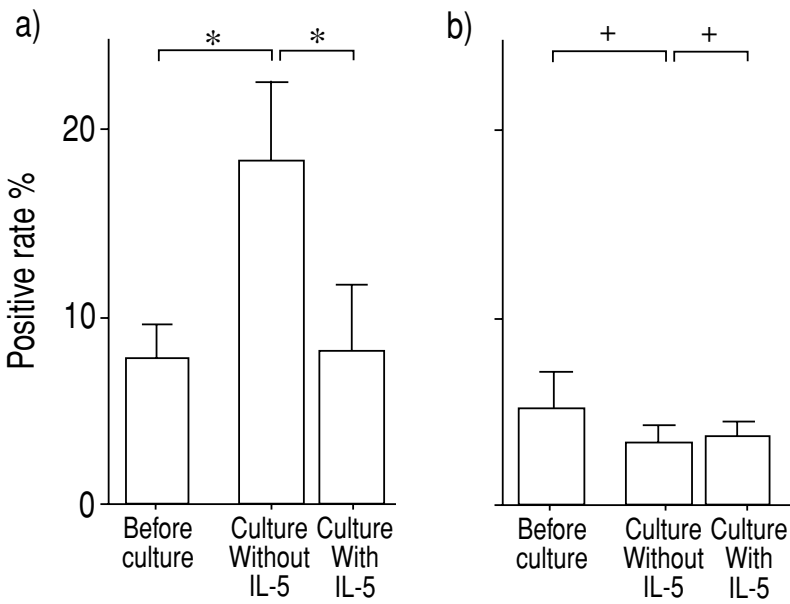

Fig. 3. - Effect of interleukin (IL)-5 on Fas expression on eosinophils from peripheral blood (PB-Eos) and eosinophils from bronchoalveolar lavage (BAL-Eos). Eosinophils from a) PB and b) BAL were cultured with or without IL-5 $\left(10 \mathrm{ng} \cdot \mathrm{mL}^{-1}\right)$ for $24 \mathrm{~h}$. Data are the mean $\pm \mathrm{SD}$ of six cases. *: $\mathrm{p}<0.05 ;+$ : not significant.

Furthermore, it was found that stimulation with antiFas antibody clearly induced apoptosis of PB-Eos but not of BAL-Eos (fig. 4). These data suggest that apoptosis of PB-Eos was related to Fas antigen, but that of BAL-Eos had little relation to Fas in CEP.

\section{Discussion}

The differences of the kinetics of apoptosis between PB-Eos and BAL-Eos in CEP were evaluated. The survival rate of the BAL-Eos after culturing for 3 days was significantly higher than that of the PB-Eos. This was probably the result of a decreased propotion of apoptotic cells in BAL-Eos compared to PB-Eos. IL-5 inhibited apoptosis in both types of cells, and prolonged 

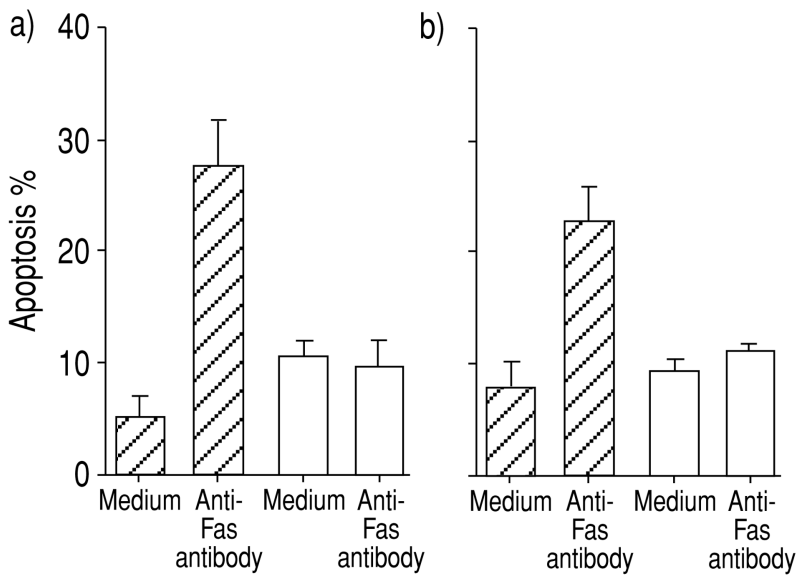

Fig. 4. - Effect of anti-Fas antibody on apoptosis of eosinophils from peripheral blood (PB-Eos) and eosinophils from bronchoalveolar lavage (BAL-Eos). $\square$ : PB-Eos and $\square$ : BAL-Eos were incubated with anti-Fas antibody $\left(\mathrm{CH} 11: 300 \mathrm{ng} \cdot \mathrm{mL}^{-1}\right)$ for $24 \mathrm{~h}$ Data from two representative cases are shown (a) and b) respectively). Data are the mean \pm SD of triplicate cultures.

their survival. Fas antigen seemed to be involved in the apoptosis of PB-Eos but not of BAL-Eos.

The anti-apoptotic effect of IL-5 has been investigated at the molecular level $[14,15]$. IL-5 has ligandspecific $\alpha$-receptor subunits and common $\beta(\beta c)$ subunits. The latter is considered the most important signalling molecule involved in propagating antiapoptotic signals. Another report concluded that the inhibitory effect of IL-5 on apoptosis was due to upregulation of bcl-2 expression [16]. Although eosinophil apoptosis was suspected to be related to Fas antigen, most reports have concluded that Fas and $\beta \mathrm{c}$ receptor were functionally independent [7, 14, 17-19]. In the present study, it was demonstrated that IL-5 downregulated the Fas expression on PB-Eos in CEP. Although the regulation of IL-5 might indirectly affect Fas expression, one of the explanations of this effect might be the presence of the heterogeneous eosinophils in CEP. A previous report about Fas expression of eosinophils from healthy donors showed that IL-5 did not affect Fas expression on eosinophils [18]. Another report showed that expression of Fas on eosinophils from atopic dermatitis (AD) patients did not change over time in culture, and was not modulated by IL-5 [19]. The authors have also found that eosinophils from $\mathrm{PB}$ of patients with other human diseases with eosinophilia, such as hypereosinophilic syndrome, bronchial asthma, and Kimura's disease, also show a significant correlation between IL-5 stimulation and Fas expression (data not shown). Although further study is needed for confirmation, this is the first description showing the possibility of a correlation between IL-5 and Fas expression on eosinophils in CEP.

It was previously shown that there is a possibility of eosinophil functional heterogeneity, through the study of the chemotactic response of eosinophils to eosinophil chemotactic factors and the expression of eosinophil surface antigen in human diseases with hypereosinophilia $[12,20,21]$. In the present study, the kinetics of apoptosis of eosinophils from CEP patients might be heterogeneous, at least compared to those from AD patients and healthy donors.

More interestingly, the expression of Fas antigen on BAL-Eos apparently did not change during the culture, although it was increased on PB-Eos (figs. 2 and 3). As shown in figure 4, exogenous addition of anti-Fas antibody (CH11) did not induce apoptosis of BAL-Eos, whereas it did induce that of PB-Eos. The mechanism of Fas resistance is not fully understood. The present difference of responsiveness might have been due to the priming of BAL-Eos with several cytokines, especially IL-5, in the pulmonary space, leading to an altered responsiveness to apoptosis. In previous reports, tissue eosinophils within inflammatory sites did not always undergo apoptosis following Fas stimulation [10, 11]. In nasal polyp tissue, Fas resistance of eosinophils was observed [10]. The authors of that study emphasized that Fas resistance had pathophysiological relevance in chronic eosinophilic disorders. Vignola et al. [11] also reported that apoptosis of eosinophils was decreased in the bronchi of bronchial asthma patients. The present findings showed that eosinophils in the alveolar space had a certain resistance to Fas-induced apoptosis. Further investigations such as signal transduction studies would be needed to elucidate the mechanisms involved in this resistance. The number of PB-Eos in the patients with CEP decreases immediately after steroid therapy, while the resolution of pulmonary infiltration is sometimes delayed. This delay of the resolution might be the result of the difference in apoptosis of PB-Eos and BAL-Eos.

In summary, the results of the present study indicate that the apoptosis of eosinophils from bronchoalveolar lavage were more resistant than that of eosinophils from peripheral blood in chronic eosinophilic pneumonia. The reduced apoptosis of eosinophils might be due to the effects of over-expression of pro-inflammatory cytokines such as interleukin-5 in the course of eosinophil accumulation in the lung. The apoptosis of eosinophils from peripheral blood was related to Fas antigen on the cell surface, whereas that of eosinophils from bronchoalveolar lavage was not. This difference might be explained by the chronic interleukin-5 stimulation leading to tachyphylaxis of eosinophils to interleukin-5 and a lower level of Fas expression of eosinophils from bronchoalveolar lavage in the alveolar space. The continuous interleukin-5 stimulation and the change of eosinophil reactivity might play a crucial role in chronic eosinophilic disorders in the pulmonary space. Further studies are needed to confirm this data and to elucidate the underlying mechanisms.

\section{References}

1. Carrington $\mathrm{CB}$, Addington WW, Goff $\mathrm{AM}$, et al. Chronic eosinophilic pneumonia. N Engl J Med 1969; 280: $787-798$.

2. Kita H, Sur S, Hunt LW, et al. Cytokine production at the site of disease in chronic eosinophilic pneumonitis. Am J Respir Crit Care Med 1996; 153: 1437-1441.

3. Hirshberg B, Kramer MR, Lotem M, et al. Chronic eosinophilic pneumonia associated with cutaneous T-cell lymphoma. Am J Hematol 1999; 60: 143-147. 
4. Tani K, Kashio M, Sano N, Nakamura Y, Ogushi F, Sone S. A case of sarcoidosis associated with chronic eosinophilic pneumonia. $J$ Med Invest 1998; 45: $131-136$.

5. Allen JN, Liao Z, Wewers MD, Altenberger EA, Moore SA, Allen ED. Detection of IL-5 and IL-1 receptor antagonist in bronchoalveolar lavage fluid in acute eosinophilic pneumonia. J Allergy Clin Immunol 1996; 97: $1366-1374$.

6. Bagley CJ, Lopez AF, Vadas MA. New frontiers for IL-5. J Allergy Clin Immunol 1997; 99: 725-728.

7. Matsumoto K, Schleimer RP, Saito H, Iikura Y, Bochner Y. Induction of apoptosis in human eosinophils by anti-Fas antibody treatment in vitro. Blood 1995; 86: $1447-1453$.

8. Luttmann W, Opfer A, Dauer E, et al. Differential regulation of CD95 (Fas/APO-1) expression in human blood eosinophils. Eur J Immunol 1998; 28 : $2057-2065$.

9. Druilhe A, Cai Z, Haile S, Chouaib S, Pretolani M. Fas-mediated apoptosis in cultured human eosinophils. Blood 1996; 87: 2822-2830.

10. Simon HU, Yousefi S, Schapowal A, Bachert C, Blaser K. Direct demonstration of delayed eosinophil apoptosis as a mechanism causing tissue eosinophilia. J Immunol 1997; 158: 3902 - 3908.

11. Vignola AM, Chanez P, Chiappara G, et al. Evaluation of apoptosis of eosinophils, macrophages and $\mathrm{T}$ lymphocytes in mucosal biopsy specimens of patients with asthma and chronic bronchitis. J Allergy Clin Immunol 1999; 103: 563-573.

12. Saita N, Hirayama S, Sugimoto M, Ando M, Hirashima M. Chemotactic heterogeneity of eosinophils in idiopathic pulmonary eosinophilia. Int Arch Allergy Appl Immunol 1995; 108: Suppl. 1, $25-$ 27.

13. Levi-Schaffer F, Temkin V, Malamud V, Feld S, Zilberman Y. Mast cells enhance eosinophil survival in vitro: role of TNF-alpha and granulocyte-macrophage colony-stimulating factor. J Immunol 1998; 160: 5554-5562.

14. Yousefi S, Hoessli DC, Blaser K, Mills GB, Simon HU. Requirement of Lyn and Syk tyrosine kinases for the prevention of apoptosis by cytokine in human eosinophils. J Exp Med 1996; 183: 1407-1414.

15. Pazdrak K, Olszewska-Pazdrak B, Stafford S, Garofalo R, Alam R. Lyn, Jak2 and Raf-1 kinases are critical for the anti-apoptotic effect of interleukin 5 , whereas only Raf-1 kinases is essential for eosinophil activation and degranulation. $J$ Exp Med 1998; 188: $421-429$.

16. Ochiai K, Kagami M, Matsumura R, Tomioka H. IL-5 but not interferon-gamma (INF- $\gamma$ ) inhibits eosinophil apoptosis by upregulation of bcl-2 expression. Clin Exp Immunol 1997; 107: 198-204.

17. Tsuyuki S, Bertrand C, Erard F, et al. Activation of the Fas receptor on the lung eosinophils leads to apoptosis and resolution of eosinophilic inflammation of the airways. J Clin Invest 1995; 96: 2924-2931.

18. Luttmann W, Opfer A, Dauer E, et al. Differential regulation of CD95 (Fas/APO-1) expression in human blood eosinophils. Eur J Immunol 1998; 28: 2057-2065.

19. Wedi B, Raap U, Kapp A. Significant delay of apoptosis and Fas resistance in eosinophils of subjects with intrinsic and extrinsic type of atopic dermatitis. Int Arch Allergy Appl Immunol 1999; 118: $234-235$.

20. Saita N, Ueno M, Yoshida M, Kimura T, Ando M, Hirashima M. Chemotactic heterogeneity of eosinophils in Kimura's disease. Int Arch Allergy Appl Immunol 1994; 104: Suppl. 1, 21 - 23.

21. Saita N, Yamanaka $\mathrm{T}$, Sugimoto $\mathrm{M}$, et al. Heterogeneity of eosinophils in chronic eosinophilic pneumonia. Int Arch Allergy Appl Immunol 1996; 111: Suppl. 1, 28-30. 